\title{
Reflets
}

Revue d'intervention sociale et communautaire

\section{Une revue qui nous ressemble - une revue qui nous rassemble}

\section{Nérée St-Amand et Marc Charron}

Volume 21, numéro 2, automne 2015

URI : https://id.erudit.org/iderudit/1035431ar

DOI : https://doi.org/10.7202/1035431ar

Aller au sommaire du numéro

Éditeur(s)

Reflets, Revue d'intervention sociale et communautaire

ISSN

1203-4576 (imprimé)

1712-8498 (numérique)

Découvrir la revue

Citer ce document

St-Amand, N. \& Charron, M. (2015). Une revue qui nous ressemble - une revue qui nous rassemble. Reflets, 21(2), 10-17. https://doi.org/10.7202/1035431ar d'utilisation que vous pouvez consulter en ligne.

https://apropos.erudit.org/fr/usagers/politique-dutilisation/ 


\section{Une revue qui nous ressemble - une revue qui nous rassemble}

Nérée St-Amand, Ph. D.

Professeur agrégé, École de service social, Université de Montréal

\section{Marc Charron}

Chercheur, Département de sociologie, Université Laurentienne

Reflets se donne des airs de fêtes! Les vingt ans d'existence de cette revue d'intervention sociale et communautaire, qui rend compte des recherches et des pratiques centrées sur les communautés de langue française vivant en milieu minoritaire, méritent en effet d'être soulignés.Au-delà de cette marque de pérennité attribuable à la témérité et au dévouement d'une équipe de direction qui a su de numéro en numéro concrétiser la réalisation de ce rêve un peu fou, Reflets témoigne de la vitalité qui anime nos communautés minoritaires. Pour ce faire, la revue a cherché à nourrir l'analyse des gens de terrain, en leur offrant une tribune leur permettant de discuter et d'échanger sur des interventions appuyées par la recherche scientifique de chercheuses et de chercheurs, et ainsi, favoriser la réflexion critique sur les problèmes et les enjeux de société. En effet, l'édition savante d'une revue comme Reflets joue un rôle important de révélateur, à la fois dans l'éveil des consciences et la définition des problématiques qui agitent les milieux minoritaires. Comme son nom l'indique, la revue se veut un outil de communication permettant aux gens vivant et intervenant dans ces milieux de se connaitre et se reconnaître. Et c'était là le but que visaient au départ ses fondatrices et fondateurs.

À l'origine, Reflets cherchait à combler une lacune, un manque de connaissance chez celles et ceux œuvrant dans ces communautés. Comme l'écrivait Carrière (Carrière, 1995,p.XI) : 
En Ontario, les intervenants et intervenantes qui œuvrent auprès de la communauté francophone dans le domaine des pratiques sociales et communautaires n'ont aucun outil de communication qui leur soit propre. La revue permettra de découvrir, d'analyser et de remettre en question l'évolution, la définition et la distribution des programmes et des services d'intervention sociales, communautaire et de santé en Ontario français.

Ce manque en question était d'autant plus criant que les processus de sécularisation pavant la voie à la modernité au Québec et ailleurs dans les autres provinces canadiennes ont connu des évolutions et des temporalités différentes, au point de donner des agencements institutionnels fort différents d'une province à l'autre.

Au cours des décennies 1960 et 1970 au Québec, on a assisté avec la "Révolution tranquille " à un travail systématique de substitution et de prise en charge par l'État des fonctions caritatives, éducatives et de service social antérieurement assumées par l'Église. Suivant la période dite de la "Grande Noirceur ", tentative ultime des élites politiques conservatrices et cléricales de maintenir les traditions contre les vents réformistes et à endiguer tant bien que mal les changements sociaux portés par les mouvements sociaux de l'époque, le processus de sécularisation a inexorablement suivi son cours. Dans les autres provinces canadiennes, le déclin de l'influence de l'Église a été moins apparent et surtout moins radical. Il en a découlé des régimes provinciaux différents de laïcité qui sont les produits de compromis politiques et inscrits dans une histoire singulière. Les cadres institutionnels qui en résultent donnent lieu à des agencements particuliers.

Ainsi, en Ontario comme ailleurs au Canada, la perte d'influence de l'Église dans la détermination des règles ne s'est pas produite au même rythme et de la même façon. Par exemple, en Ontario, on a maintenu un système scolaire confessionnel parallèle à un système public. Cette mixité, confessionnelle et publique, 
caractérise encore aujourd'hui bon nombre des institutions d'intervention sociale, communautaire et de santé. Il en va de même des Sociétés d'aide à l'enfance qui, bien qu'assujetties à une réglementation et un financement publics, demeurent des organismes privés d'origine confessionnelle. Ainsi, les institutions des autres provinces canadiennes ne correspondaient pas aux transformations institutionnelles du Québec, compliquant d'autant l'identification et le transfert de connaissances des pratiques documentées et analysées par les revues francophones québécoises phares en service social. Par ailleurs, la situation minoritaire des francophones à l'extérieur du Québec obligeait ces derniers à transiger avec un État provincial et des organismes sociaux et communautaires où l'anglais est la langue principale de communication, ce qui n'était manifestement pas le cas au Québec. Le besoin d'un outil de communication bien à nous, qui nous ressemble et nous rassemble, en favorisant le réseautage entre francophones et l'accès à des services en français, était donc ressenti fortement.

Qui plus est, en raison des différences institutionnelles, les chercheuses et chercheurs en provenance de l'extérieur du Québec avaient un accès limité aux publications scientifiques québécoises en service social, telles que Service social et Nouvelles pratiques sociales. À l'exception de ses premiers numéros de la revue et jusqu'au tournant des années 2000 , cette dernière incluait une rubrique Pratiques d'ailleurs qui portait sur les pratiques des autres provinces ou d'ailleurs dans le monde. Mais progressivement, il y a eu centration sur l'espace québécois, rendant encore plus pressant le besoin d'une tribune à nous pour faire état de nos recherches et de nos pratiques.

Dans un tel contexte, le numéro de Reflets que vous feuilletez actuellement relève tout un défi. En plus de présenter un bilan du travail accompli depuis son lancement en 1995, ce numéro spécial vise à cerner l'impact de la revue sur les différentes ressources, tant en santé qu'en service social. En plus, il porte son regard sur l'avenir en tentant de définir le cap à privilégier à compter de maintenant.Vous constaterez que les différents textes répondent à cette question et suggèrent de riches pistes de réflexion tout 
en proposant des formes d'intervention adaptées aux réalités des personnes vivant et travaillant en contexte francophone minoritaire.

Les lecteurs et lectrices de la version imprimée auront sans nul doute remarqué notre page couverture spéciale, cette toile qui aborde la thématique de l'envol, de la prise de pouvoir, est signée par une artiste de la relève. Déjà, le ton est donné : Reflets a des ailes, elle est sortie de son nid, et elle est en plein vol!

C'est d'ailleurs à cet exercice récapitulatif que se prêtent Molgat et Trahan-Perreault en dressant le bilan de la recherche en travail social dans les communautés minoritaires au Canada. De cet examen des thématiques qui dominent les préoccupations des chercheuses et chercheurs, émergent une grande cohérence d'ensemble visant la "complétude institutionnelle ", pour reprendre l'expression de Breton (1985), et une plus grande maîtrise et autonomie, notamment dans le secteur de la santé et en matière de violence faite aux femmes.

Molgat et Trahan-Perreault abordent ces deux thématiques en établissant un parallèle avec les luttes scolaires passées; cellesci avaient pour moteurs le maintien et l'épanouissement de la communauté franco-ontarienne et ont eu pour résultat la gestion par celle-ci de ses propres écoles. Les auteurs rappellent que dans les domaines de la santé et de la violence faite aux femmes, la douleur et la souffrance ne s'expriment pleinement que dans sa langue maternelle et ils mettent en évidence les carences des services dits «bilingues " destinés aux communautés de langue française.

À l'heure des bilans, l'article de Marin et St-Amand revient sur les vingt ans de Reflets. Les auteurs en retracent l'origine, le contexte dans lequel la revue a fait son apparition, mettent en lumière les principaux thèmes, font une brève incartade du côté de la présentation et présentent son lectorat comme une illustration de sa pertinence sociale. La multiplicité des perspectives ressortant de cette revue de la littérature souligne la richesse des thèmes abordés et reflète le dynamisme des auteures et auteurs des différents articles publiés à ce jour. Les problématiques de la 
violence, des inégalités ou de la santé, entre autres, deviennent plus complexes en contexte minoritaire. Marin et St-Amand déplorent cependant l'absence de certains thèmes et problématiques sociales qui auraient mérité un approfondissement, par exemple, la pauvreté et les populations autochtones.

Toujours à l'heure des bilans, l'Entrevue est le résultat d'une écriture à six mains; d'anciens et d'actuels directeurs et directrices de Reflets nous font part du chemin parcouru par la revue et en explorent des avenues possibles. À l'instar de Molgat et TrahanPerreault, ces trois signataires font état de faits marquants et témoignent de l'impact de Reflets tant sur le plan de la formation que sur ceux de la pratique et de la recherche.

Chacun à sa façon, trois autres articles du Dossier reflètent la réalité de Francophones vivant en milieu minoritaire. D'abord, van Kemenade, Bouchard et Bergeron abordent les enjeux liés à la santé des aînés francophones au Canada. En raison $\mathrm{du}$ vieillissement prononcé des populations vivant en milieu minoritaire et où les femmes ont une espérance de vie plus grande que celle des hommes, cela entraîne des disparités entre les sexes. Notamment, les femmes sont sujettes à des conditions précaires de par la faiblesse de leur revenu et de leur niveau d'éducation. La plupart vivant en milieux ruraux, elles éprouvent plus durement l'absence en français des soins à domicile dont elles ont besoin en raison surtout de la réduction de mobilité qui accompagne malheureusement leur avancée en âge. Cette analyse différenciée selon le genre nous rappelle l'importance de considérer les liens entre les différents facteurs structurels, pour une meilleure compréhension des réalités de vieillir en milieu minoritaire.

Puis, Lirette, Kabano, Gauthier et Beaton traitent des perceptions du marché du travail au Nouveau-Brunswick des personnes ayant un handicap. Un constat s'impose. Malgré les diverses législations visant à mieux intégrer les personnes ayant des limitations fonctionnelles, le jeu des perceptions réciproques entre employeurs et employées ou employés futurs ou actuels constitue un frein à leur intégration. Tout en faisant valoir l'importance des droits à l'égalité, cet article devrait interpeler tout autant les 
employeurs que le monde de l'intervention pour une meilleure compréhension des défis importants que vivent les personnes qui ont un handicap et qui sont à la recherche d'un emploi.

Enfin, Delaquis s'intéresse à la construction de la masculinité de jeunes franco-manitobains. Somme toute, les dires de ces jeunes semblent assez imperméables aux discours invitant à une remise en question des normes de la masculinité traditionnelle. L'incarnation de ce modèle où l'image du sportif, du rebelle à l'autorité, semble servir de référence et demeure aussi populaire auprès des adolescents franco-manitobains pour se définir nous rend perplexes. De plus, la figure de l'artiste qui pourrait inviter à une redéfinition de ce concept semble agir en sens inverse, comme repoussoir. Un tel constat tend à indiquer une transformation des mentalités tardant à venir. Est-ce là un effet du milieu, voire d'un manque de diffusion d'un discours urbain, avant-gardiste qui bouscule et remet en question la masculinité traditionnelle en invitant au changement? Nous ne saurions le dire, mais il souligne l'importance d'offrir aux jeunes francophones des modèles alternatifs. Si cet article reflète la réalité des jeunes hommes en contexte minoritaire, la question se pose à savoir jusqu'à quel point il est possible de dévier des normes plutôt rigides de ce qu'impose ce modèle dominant, sans parler des difficiles marges de manœuvre en terme de résistance.

Du côté des pratiques, Garceau et Marcocci partagent l'évaluation du programme de formation à distance de l'Université Laurentienne. Cette formation en service social s'avère importante, car elle permet de rejoindre et de transmettre aux francophones éparpillés à travers le Canada, un savoir professionnel de haute qualité, comme en témoigne la réception très favorable des étudiantes et étudiants. Il est aussi question de formation dans l'article de Levesque, Cayouette-Marsh et Turpin, puisque la Semaine nationale du travail social à l'Université d'Ottawa est l'occasion d'offrir un lieu de discussion, de réflexions autour de pratiques engagées, empreintes de justice sociale, de défense des droits humains et d'analyse structurelle de la société en vue d'un changement social à la faveur des plus défavorisés de nos sociétés. 
Là encore, l'appréciation des étudiantes et des étudiants ne tarit pas d'éloges, car cet événement leur permet d'établir de forts liens théorie et pratique.

Pour sa part, Perron Roach rappelle l'importance de l'Hôpital Montfort comme symbole des luttes franco-ontariennes sur la voie de l'autodétermination et de la maitrise de ses propres institutions. Afin que ce symbole demeure toujours vibrant, l'Hôpital Montfort doit évoluer au rythme des transformations qui traversent une société franco-ontarienne balayée par l'apport de nouveaux arrivants provenant d'horizons culturels divers. De plus, en raison de sa désignation par le gouvernement ontarien de centre hospitalier universitaire, groupe A, sa mission de formation de professionnelles ou professionnels francophones dans le domaine de la santé et dans celui du développement de la recherche sur la santé des Ontaroises et Ontarois s'en trouve considérablement élargie.

Enfin, à titre de porte-parole de la Table ronde des organismes volontaires d'éducation populaire de l'Outaouais (TROVEPO), Greason invite le lectorat à recentrer les pratiques communautaires sur les droits humains et à ne pas perdre de vue la personne ou les groupes de personnes que l'on accueille. À partir de termes tels que " clients/clientes ", " usagers/usagères ", " consommateurs/ consommatrices ", Greason dénonce l'approche comptable instillée par un néolibéralisme sournois chez les intervenantes et intervenants qui en viennent à oublier la personne et le collectif dont ils doivent prendre la défense. Entrevoyant la pratique communautaire comme un lieu d'intervention favorisant la liberté, l'égalité et la solidarité, les organismes doivent reconnaitre la personne comme citoyenne, comme une actrice pleine et entière.

En somme, un menu riche et diversifié pour un $20^{\mathrm{e}}$ anniversaire à souligner! Bonne lecture! 


\section{Bibliographie}

BRETON, Raymond (1985). "L'intégration des francophones hors Québec dans des communautés de langue française ", Revue de l'Université d'Ottawa,Vol. 52, No 2, p.77-99.

CARRIÈRE, Richard (1995). "Présentation - Reflets : revue ontaroise d'intervention sociale et communautaire », Reflets : revue ontaroise d'intervention sociale et communautaire,Vol. 1, No 1, p. I-XI. 